In Crescendo. Institucional. 2015; 6(1): 207-223

\title{
EFECTOS DE UN PROGRAMA DE ENTRENAMIENTO DE FUERZA MUSCULAR SOBRE LOS NIVELES DE GLUCOSA EN LA SANGRE EN ADULTOS SEXAGENARIOS
}

\section{EFFECTS OF A MUSCLE STRENGTH TRAINING PROGRAM ON GLUCOSE LEVELS BLOOD IN ADULTS SIXTIES}

\author{
Mendieta Toledo, L. B. ${ }^{1}$ \\ Mendieta Toledo, L. R. ${ }^{2}$ \\ Chamba Zambrano, J. M. ${ }^{3}$
}

\section{RESUMEN}

El objetivo de este trabajo es conocer los efectos de un programa de entrenamiento de la fuerza muscular sobre los niveles de glucosa en la sangre en adultos sexagenarios de Santo Domingo de los Tsáchilas, Ecuador. En cuanto a los materiales y método, se evaluaron a 44 participantes, distribuidos de manera aleatoria en dos grupos (ensayo controlado aleatorizado ECA), 0 (grupo control) $(\mathrm{n}=22)$ con actividades de la vida diaria y 1 (grupo entreno) ( $\mathrm{n}=22)$ con entrenamiento basado en la fuerza muscular. Se evaluó antes y después de la intervención con pruebas clínicas y físicas para determinar como valoración principal la glucosa en la sangre. Se realizó una revisión médica de los pacientes antes del programa y un monitoreo durante aquel. Respecto a los resultados, las pruebas para el grupo entreno fueron disminución de los niveles de glucosa en la sangre de un 6,36 \%. En el grupo control no existieron cambios significativos. Las conclusiones que se obtuvieron fueron que con un programa de entrenamiento de la fuerza muscular, los niveles de glucosa en sangre disminuyeron en los adultos sexagenarios participantes.

Palabras claves: glucosa en la sangre, fuerza muscular, adultos sexagenarios.

1 Profesor de educación básica, licenciado en Educación Física, magíster en Actividad Física y Salud, docencia universitaria e investigación educativa, profesor de natación (Real Federación Española de Natación), docente e investigador de la Pontificia Universidad Católica del Ecuador (Pucesd).

2 Profesor de natación, entrenador de actividad física, investigador de la Universidad Técnica de Loja (UTPL).

3 Profesor de educación básica, licenciado en Ciencias de la Educación, mención en inglés, docente e investigador de la Universidad Técnica de Machala (Utmach). 


\begin{abstract}
Objective: To determine the effects of a muscle strength training program on blood glucose levels in people in their sixties from Santo Domingo de los Tsáchilas-Ecuador. Materials and Methods: Forty-four participants were evaluated and randomly divided into two groups (randomized controlled trial RCT), 0 (control group) $(\mathrm{n}=22)$ with daily living activities and 1 (trained group) $(n=22)$ with training based on the muscle strength. It was assessed before and after intervention with clinic and physical tests to determine as primary outcome, measure blood glucose. A medical examination of patients was conducted before the program and a monitoring during treatment. Results: The results obtained in the tests for the train group were: decreased levels of blood glucose $\pm 6,36 \%$. In the control group there were no significant changes. Conclusions: The conclusions obtained are as follows: with a training program of muscle strength, blood glucose levels, decreased in people in their sixties.
\end{abstract}

Keywords: blood glucose, muscle strength, people in their sixties. 


\section{INTRODUCCIÓN}

Estudios realizados acerca de la acción de los trabajos aeróbicos y anaeróbicos sobre la actividad de la insulina (transportadora de la glucosa por la sangre) durante el entrenamiento y posentrenamiento (14 días después) reflejan una mejoría de hasta el 52 \% de la sensibilidad a la insulina hasta 14 días después del entrenamiento (1).

El entrenamiento de la fuerza muscular produce efectos de disminución en los niveles de glucosa en la sangre en los pacientes sometidos a programas entreno, y la práctica de ejercicio de alta intensidad puede conducir a mejoras similares en la aptitud física y la función física en las personas con diabetes tipo 2 (2). Mas no se han determinado mejorías en los niveles de glucosa en la sangre de los mismos participantes.

El músculo mejora en varios predictores que al final reflejan un progreso en el estado de su salud. Los avances en el estado de salud con el tratamiento de programas de fuerza muscular es una realidad; un método eficaz y barato capaz de combatir enfermedades propias de los adultos para aliviar su salud y, por ende, mejorar su calidad de vida.

La fisiología del ejercicio se atreve hoy a enunciar la teoría que el músculo es un verdadero órgano endocrino, lo cual conlleva a la responsabilidad de realizar un mayor número de estudios de actividad física en sus dimensiones pertinentes.

En la investigación realizada en la ciudad de Loja, Ecuador, se evidencian mejorías de algunos predictores de salud, como la condición física y la fuerza muscular (3); sin embargo, no se habían realizado estudios sobre glucosa en la sangre.

\section{Objetivos}

Conocer los efectos de un programa de entrenamiento de la fuerza muscular sobre los niveles de glucosa en la sangre en adultos sexagenarios de Santo Domingo de los Tsáchilas-Ecuador.

\section{MATERIALES Y MÉTODOS}

Se evaluó a 44 participantes, distribuidos de manera aleatoria en dos grupos mediante un ECA, cero (grupo control) $(\mathrm{n}=22)$ con actividades de la vida diaria y un (grupo entreno) $(\mathrm{n}=22)$ con entrenamiento basado en la fuerza muscular. Se evaluó antes y después de la intervención, se realizó un examen clínico en laboratorio para determinar los niveles 
de glucosa antes de empezar el programa entreno, y se sometió a pruebas físicas para determinar la prescripción médica del ejercicio. Se solicitó un consentimiento informado por escrito de los participantes, y se realizó una revisión médica de los pacientes antes del programa y un monitoreo durante aquel.

Los métodos empleados para determinar la prescripción médica del ejercicio de los participantes del estudio fueron pruebas de FCR y FCM, así como la medición de parámetros físicos (ficha bioantropométrica) para el peso y el IMC. Pruebas clínicas sobre glicemia.

Se trabajó un ensayo controlado aleatorio (ECA, ofic. 10-12-2014). El comité ético de investigaciones de la Pontificia Universidad Católica del Ecuador, sede Santo Domingo (Santo Domingo, Ecuador, Investigium 2014-02) aprobó el diseño, los protocolos y el procedimiento de consentimiento informado por escrito, y todos los participantes proporcionaron su aprobación de manera formal. Después de las medidas realizadas al inicio del estudio, los participantes fueron asignados al azar a los grupos de control o de intervención, como se explica más adelante. Los dos grupos fueron evaluados antes de empezar el programa y de su designación. El grupo de intervención continuó un programa de entrenamiento de la fuerza muscular de 12 semanas (más una semana de ambientación y una semana de preevaluación), que fue seguido por una semana de evaluación posprograma por los mismos investigadores que realizaron las medidas iniciales del estudio. El trabajo se hizo entre diciembre del 2014 y marzo del 2015, para lo cual se aplicaron las normas éticas de la Declaración de Helsinki, modificadas por última vez en la 64. ${ }^{a}$ Asamblea General, Fortaleza, Brasil, octubre del 2013.

\section{Participantes en el estudio y criterios de selección}

Los potenciales participantes fueron 58 sexagenarios residentes en la comunidad de Santo Domingo de los Tsáchilas-Ecuador, seleccionados del centro ambulatorio de la seguridad social del Ecuador, en Santo Domingo. A todos ellos se les practicó un reconocimiento médico completo, y el profesional que los diagnosticó les extendió un certificado médico. Los criterios de inclusión fueron los siguientes: 60 años o más, no tener previsto trasladarse de la ciudad durante el estudio, capacidad de caminar sin ayuda, capacidad de comunicarse, y capacidad y voluntad de proporcionar una autorización informada. Los criterios de exclusión fueron los siguientes: enfermedad aguda o terminal, infarto de miocardio en los últimos tres meses, incapacidad de caminar, padecer enfermedad cardiovascular inestable u otra afección médica, fractura en las extremidades superiores o inferiores durante los últimos tres meses, demencia grave, falta de voluntad para cumplir con los requisitos del estudio o para ser asignados al azar al grupo de control o de entrenamiento, enfermedad neuromuscular o consumir fármacos que afecten a la función neuromuscular. Se emitió un certificado médico de monitoreo durante el programa y una prescripción médica del ejercicio. 


\section{Aleatorización y bloqueo}

El encargado del diseño estadístico asignó al azar a los participantes al grupo de control o de entrenamiento, con un diseño de bloqueo para sexo, según una secuencia de aleatorización generada por el ordenador de la marca HP.

El grupo de investigación desconocía la codificación utilizada para asignar a los participantes a los grupos ( 0 para los cuidados habituales y 1 para la intervención). El personal de evaluación también desconocía la asignación al azar de los participantes. Además se informó explícitamente a los participantes y se les recordó que no hablaran sobre su asignación al azar con el personal de evaluación. No fue posible ocultar la asignación de grupos al personal que participó en el entrenamiento.

Finalmente iniciaron el estudio 44 participantes: 22 para cada grupo (anexo un diagrama de flujo). Los grupos eran homogéneos inicialmente para los principales datos demográficos, médicos y funcionales ( $p>0,05$ para todas las comparaciones entre grupos).

\section{Grupo con cuidados habituales}

Durante el estudio, los participantes del grupo control (cuidados habituales) siguieron los consejos generales de los entrenadores de realizar actividades de la vida diaria y los posibles efectos de los ejercicios de movilidad. Ellos hicieron movimientos pasivos y de poca actividad de 40 a 45 minutos al día (cinco días a la semana) consistentes en una serie de estiramientos suaves y rítmicos de las articulaciones individuales.

\section{Grupo de intervención}

Los participantes asignados al grupo de intervención siguieron un plan de cinco sesiones semanales de entrenamiento de la fuerza muscular de forma consecutiva durante 12 semanas (60 sesiones en total). Cada sesión duró aproximadamente de 45 a 50 minutos. El programa de los ejercicios se centró en el entrenamiento de la fuerza muscular, cada sesión comenzó y terminó, respectivamente, con periodos de calentamiento y enfriamiento de baja intensidad (aproximadamente de cinco a siete minutos cada uno), que consistieron principalmente en ejercicios de estiramiento con implicación de todos los grupos musculares. La parte central de la sesión consistió en entrenamiento para el fortalecimiento muscular (hipertrofia) con implicación de los grandes grupos musculares de las extremidades inferiores y superiores, es decir, ejercicio de fuerza de presión de las piernas y brazos realizado con máquinas de resistencia variable de fabricación artesanal. Los participantes realizaron entre dos y tres series de ocho a diez repeticiones con periodos de descanso de uno a dos minutos entre ejercicios.

Se trabajó un programa piramidal ascendente con carga desde el 70 \% y una repetición máxima (1RM) al inicio del programa hasta el 100 \% de 1RM al final. Los ejercicios de estiramiento de los músculos implicados en los ejercicios anteriores se hicieron durante los periodos de descanso. Se aconsejó a los participantes que efectuaran todos los 
movimientos de forma lenta y controlada, y que no contuvieran la respiración durante el ejercicio.

\section{Familiarización y evaluación de la fiabilidad}

Antes de comenzar el estudio, los participantes hicieron un periodo de ambientación de una semana con las pruebas descritas más abajo, que consistieron en sesiones de aproximadamente 45 minutos. Cada sesión estuvo precedida por un calentamiento, y terminó con un enfriamiento que consistió en las mismas actividades y la misma duración que el periodo de entrenamiento. Cada sesión de ambientación consistió en una o dos series de seis a ocho repeticiones de los ejercicios. También se evaluó y reevaluó la fiabilidad de cada criterio de valoración. Se obtuvo un alto coeficiente de correlación intraclase ( $\mathrm{R}$ consistentemente $>0,98 ; \mathrm{p}<0,001$ ) entre las pruebas repetidas para todas las pruebas estudiadas.

\section{Variables principales:}

\section{Niveles de glucosa en sangre}

Se analizaron los niveles de glucosa en las personas sometidas al programa en dos ocasiones, antes de empezar el programa y una vez finalizado, a través de pruebas clínicas de glicemia para determinar los niveles de glucosa.

\section{Variables secundarias:}

\section{Fuerza de piernas}

Se evaluó la fuerza muscular dinámica de las extremidades inferiores mediante un protocolo normalizado de la prueba con fuerza de presión de las piernas sentado con un máximo de seis a siete repeticiones (de seis a siete RM). El valor de una RM se estimó utilizando la ecuación de Brzycki: 1RM=102,78-2,78 x número de repeticiones. Las cargas iniciales fueron de $70 \%$ al $100 \%$ del peso corporal. Después de un breve periodo de descanso (dos minutos) se añadieron incrementos de $4 \mathrm{~kg}$ a $6 \mathrm{~kg}$ hasta que el participante fuera incapaz de levantar la carga más de seis o siete veces, lo cual en general ocurrió después de cinco ensayos. Todos los participantes pudieron levantar la carga inicial al menos una vez. Para lograrlo se les enseñó la forma adecuada de respirar para cada movimiento y para levantar el peso.

\section{Fuerza de brazos}

Se evaluó la fuerza muscular dinámica de las extremidades superiores mediante un protocolo normalizado de la prueba con fuerza de presión de los brazos acostado con un máximo de seis a siete repeticiones (de seis a siete RM). El valor de una RM se estimó utilizando la ecuación de Brzycki: $1 \mathrm{RM}=102,78-2,78$ x número de repeticiones. Las cargas 
iniciales fueron de $70 \%$ al $100 \%$ del peso corporal. Después de un breve periodo de descanso (dos minutos) se añadieron incrementos de $2 \mathrm{~kg}$ a $4 \mathrm{~kg}$ hasta que el participante fuera incapaz de levantar la carga más de seis o siete veces, lo cual en general ocurrió después de cinco ensayos. Todos los participantes pudieron levantar la carga inicial al menos una vez. Para lograrlo se les enseñó la forma adecuada de respirar para cada movimiento y para levantar el peso.

\section{Análisis estadístico}

Para las comparaciones iniciales entre grupos (cuidados habituales frente a intervención), y para comparar la glucosa y fuerza muscular se analizaron las variables continuas con la prueba $\mathrm{T}$ de Student para datos no apareados (o su equivalente no paramétrico, la prueba U de Mann Whitney) y pruebas de chi ${ }^{2}$ para datos nominales. Las comparaciones múltiples se ajustaron para evitar la significación masiva.

Para evaluar los efectos del entrenamiento sobre las variables del estudio de la glucosa y fuerza muscular se analizaron los datos según el principio de intención de tratar. Cuando no se contaron con datos posteriores a la prueba, se consideraron las puntuaciones obtenidas al inicio del estudio, así como las de después de la prueba. Además se utilizó un análisis de varianza (Anova) bifactorial (grupo y tiempo) con medidas repetidas.

El análisis se repitió utilizando los valores iniciales como covariables. Para cada variable dependiente se informó del nivel de significación correspondiente a los efectos del grupo principal (entre sujetos), del tiempo (para cada sujeto) y de la interacción (grupo $\mathrm{x}$ tiempo). Con el fin de evitar un error tipo I se realizaron comparaciones post hoc (comparaciones previas y posteriores en cada grupo mediante la prueba de Bonferroni) solo cuando se obtuvo una interacción significativa. Para todos los criterios de valoración se hicieron pruebas de Anova para los dos grupos enteros. Asimismo, el nivel de significación de la prueba se estableció en 0,05.

\section{Cumplimiento y efectos secundarios}

La fidelidad del programa presentó hasta un 100 \% las evaluaciones posprograma, ya que todos los participantes culminaron el estudio. No se observó ningún efecto adverso ni problema de salud atribuible al entrenamiento indicado en ninguno de los participantes del grupo de intervención. Un participante padecía de dolor en la región lumbar transitoria al inicio del programa de entrenamiento y, por tanto, tuvo que reanudarlo con una carga menor (50 \% 1RM), lo cual le permitió terminar la intervención de manera satisfactoria.

\section{Resultados principales}

Se encontró efecto significativo del grupo, del tiempo o de la interacción $(\mathrm{p}=0,05)$ para la glucosa en el análisis de sangre. Mientras en el grupo control durante la prueba no se 
obtuvieron datos estadísticamente significativos, en el grupo entreno disminuyeron los niveles de glucosa en la posevaluación en un $\pm 6,36$ \% (tabla y figura 1).

\section{Resultados secundarios}

No se observó efecto significativo del grupo ni del tiempo ( $>0,05)$, pero se observó un efecto significativo de la interacción grupo por tiempo $(\mathrm{p}=0,018)$ para la prueba de fuerza de presión de las piernas 1RM (tabla y figura 2) y prueba de fuerza de brazos (tabla y figura 3). En el grupo de intervención, la fuerza de presión de las piernas aumentó significativamente $\pm 32 \%$ con el entrenamiento y en el grupo control aumentó un $\pm 4,04 \%$; la fuerza de brazos aumentó $\pm 18,26 \%$ en el grupo entreno y en el grupo control aumentó un $\pm 4,34$, aunque no se alcanzó la significación estadística en las comparaciones post hoc dentro de este grupo ( $\mathrm{p}=>0,05)$.

\section{DISCUSIÓN}

Siguiendo los estudios de López J. (2015), se puede inferir que la disminución de glucosa en la sangre se debe a la regulación de la insulina por la acción de programas de entrenamiento de la fuerza. Taylor J., Fletcher J., Mathis R. y Cade W. (2014) indican que, al igual que los resultados del presente estudio, la práctica de actividad física de alta intensidad produce mejoría en pacientes con diabetes tipo 2 en cuanto a la condición física, mas no reflejan las mejorías en los niveles de glucosa en la sangre de los mismos participantes. Mendieta L. (2013), en el estudio realizado en la ciudad de Loja, evidenció mejorías de algunos predictores de salud tanto de la condición física como de la fuerza muscular, mas no analizó los niveles de glucosa en la sangre después de un programa de entrenamiento de la fuerza muscular.

\section{CONCLUSIONES}

Se concluye que el programa de entrenamiento de la fuerza muscular de 12 semanas de duración produjo efectos significativos en la reducción de los niveles de glucosa en sangre en los adultos sexagenarios que participaron en el programa; además también aumentó la fuerza muscular de las extremidades superiores e inferiores. Las recomendaciones de la presente investigación es realizar un estudio de mayor duración y con un número mayor de participantes. 


\section{REFERENCIAS BIBLIOGRÁFICAS}

López J. Entrenamiento combinado de fuerza y resistencia aeróbica y sensibilidad a la insulina; 2015. Disponible en: http://fisiologiadelejercicio.com/ actualizaciones-fisiologia-del-ejercicio/revisiones.

Taylor J., Fletcher J., Mathis R. Cade W. Effects of moderate-versus high-intensity exercise training on physical fitness and physical function in people with type 2 diabetes: a randomized clinical trial; 2014. Disponible en: http://www.ncbi.nlm.nih.gov/ pubmed/25082918L

Mendieta L., Yaguachi F., Toledo K. Programa de entrenamiento para el incremento de la fuerza muscular; 2013. Disponible en: http://www.uap.edu.pe/Investigaciones/Esp/ Revista_16-02_Esp_05.pdf

\section{FIGURAS Y TABLAS}

Tabla 1. Glucosa en sangre

\begin{tabular}{ccc}
\hline Variables & preprograma & posprograma \\
\hline Entreno & 144,18 & 135 \\
\hline Control & 144,18 & 142 \\
\hline
\end{tabular}

Figura 1. Niveles de glucosa en sangre

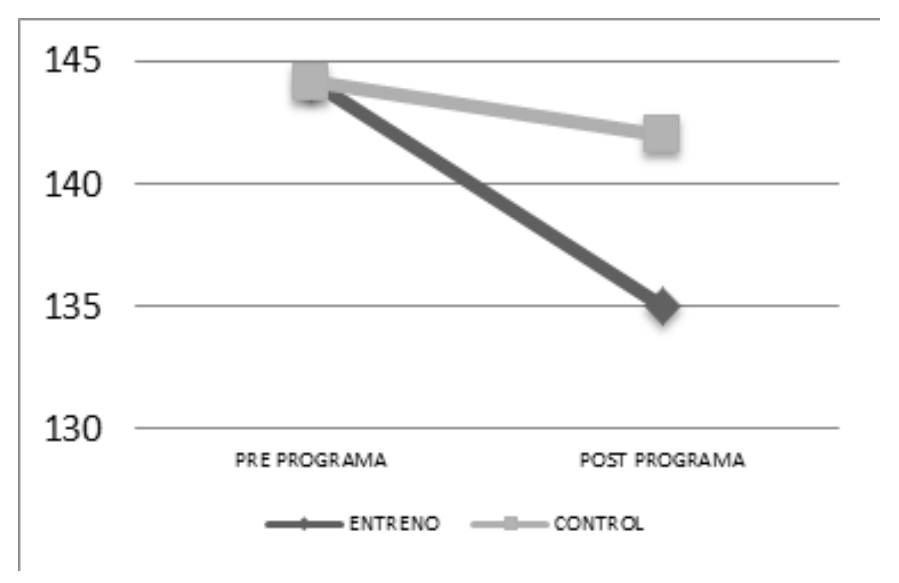


Tabla 2. Fuerza de piernas

\begin{tabular}{ccc}
\hline Variables & Preprograma & Posprograma \\
\hline Control & 81,00 & 84,50 \\
\hline Entreno & 79,29 & 86,87 \\
\hline
\end{tabular}

Figura 2. Fuerza de piernas

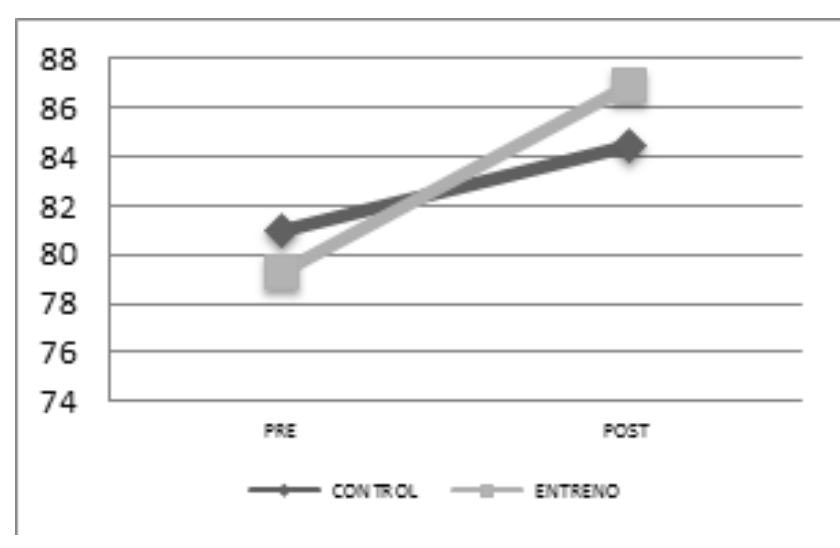

Tabla 3. Fuerza de brazos

\begin{tabular}{ccc}
\hline Variables & Preprograma & Posprograma \\
\hline Peso kg control & 57,50 & 60,00 \\
\hline Peso kg entreno & 57,50 & 68,00 \\
\hline
\end{tabular}

Figura 3. Fuerza de brazos

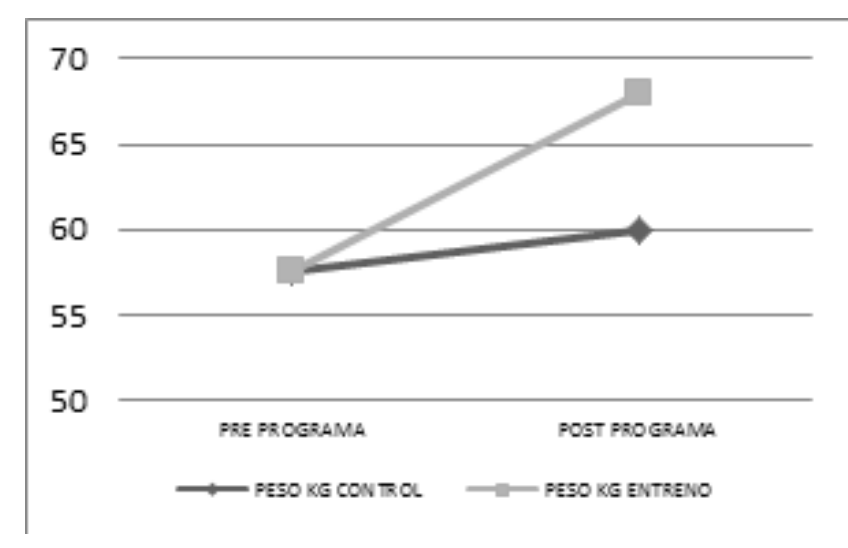


ANEXOS

Anexo 1. Consentimiento informado por escrito

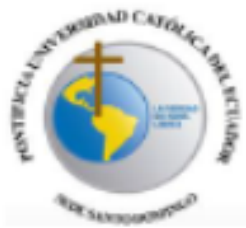

PONTIFICIA UNIVERSIDAD CATÓLICA DEL ECUADOR

Entrenamiento de la fuerza muscular más la función cardiovascular para mejoramiento de la salud en adultos de Santo Domingo

\section{CONSENTIMIENTO INFORMADO POR ESCRITO}

SANTO DOMNGO, a de 20

Yo que acepto participar en las sesiones de Entrenamiento de la fuerza muscular mas la Nuncion cardiovascular que se

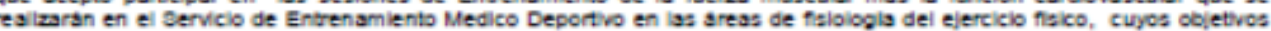
conalaten en:

- Mejorar Nuerza muacular

- Mejorar ei rendimiento aerobico y mejorar la capacidad anaerobica

- Mejorar la salud

- Comparar las ganancias con otro grupo etario del miamo centro

Estoy consciente de que los procedimientos, pruebas y tratamientos para lograr los objetvos mencionados conalstran en:

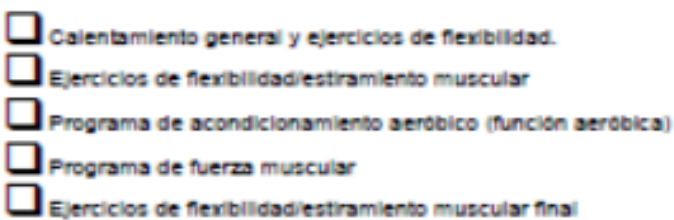

Los respos en caso de que exiatan, en mi persons inherente a las pruebas que se realzaran, me tueron explcados verpaimente, los entendl en au totaldad y los actepto de conformidad.

Es de mi conocimiento que sere ibre de retirarme del programa de ejercicio en el momento que yo ast lo desee. Tambien que puedo solicitar informacion adicional acerca de los nespos y beneficios de mi participacion en el programa. En caso de que decidera retrarme, la atencion que como paciente recibo en eata insthucion no se vera atectada.

Nombre

Firma

Direoolon____ Feoha

Fima de padre o tutor (on oace de cer mener de odad)

Tolstonoc

Toctigo___ Firma____ Direoolon 
Anexo 2. Diagrama de flujo

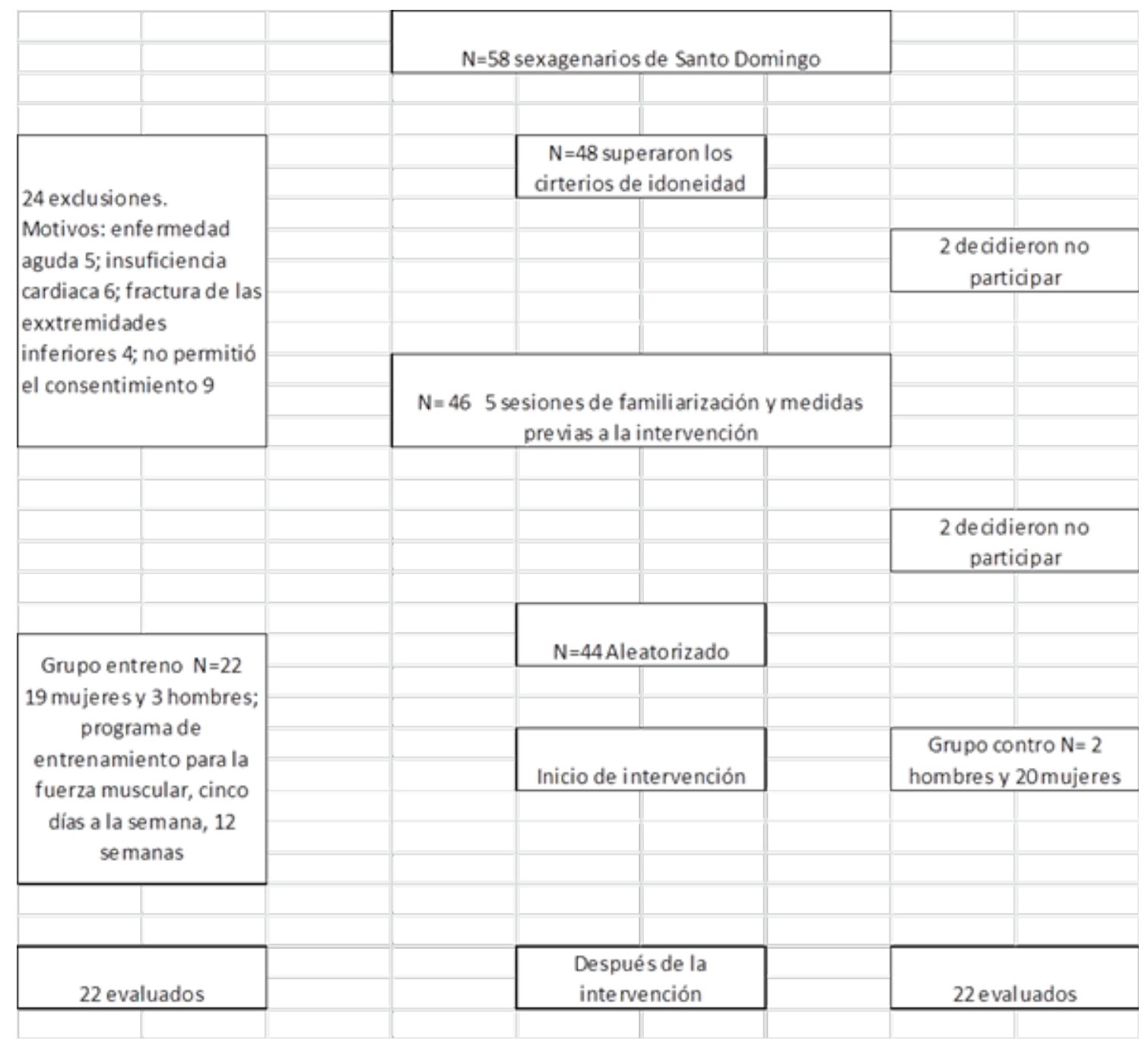


Anexo 3. Certificados

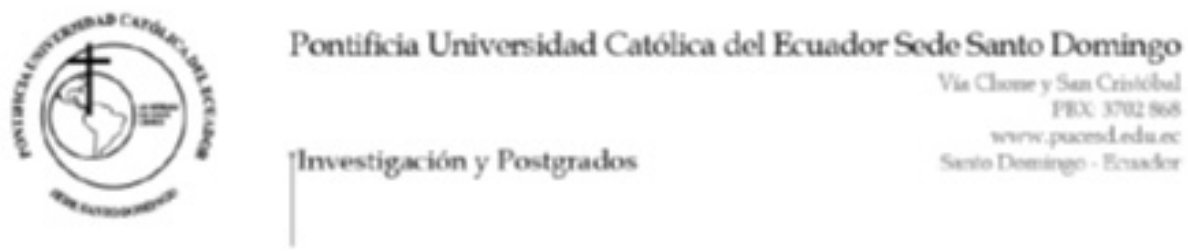

CERTIFICADO MÉDICO PARA INVESTIGACIÓN

El suscrito médico general. Alicia Fernanda Yaguachi Orellana, con COD MSP 37 FOLIO 58 N175.

\section{Certifica}

Que habiendo practicado un reconocimiento médico el dia jueves veinte y siete de noviembre de dos mil catorce (27/11/2014) al paciente denominado número uno (1), lo encontré: en buen estado de salud fisica sin defectos ni anomalias en el aparato locomotor, presenta una integra capacidad cognitiva, agudeza visual y auditiva; aparatos cardiovascular y respiratorio en optimas condiciones; el examen clíico y antropometrico revelaron altos indices de LDL, colesterol general, glicemia y tension arterial, asi como sobrepeso.

Por lo anterior se hace constar que el paciente, puede participar del programa de actividad fisica recomendado por el maestro Lenin Mendieta Toledo.

El presente certificado médico es expedido a petición del programa de investigación para establecer los efectos del mismo sobre el estado de salud de los participantes

Es cuanto puedo certificar en honor a la verdad y autorizo a los investigadores hacer uso del presente para los fines pertinentes.

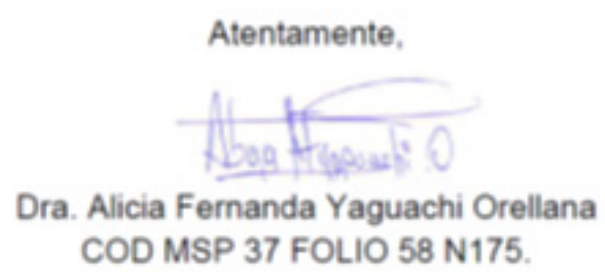

Santo Domingo, 27 de marzo de 2015 


\section{CERTIFICADO PARA INVESTIGACIÓN}

Dra. Alicia Fernanda Yaguachi Orellana, con COD MSP 37 FOLIO 58 N175. Certifico haber monitoreado el estudio 'Entrenamiento de la fuerza muscular más la función cardiovascular para mejoramiento de la salud en adultos mayores del Hospital de Santo Domingo', que se realizó en la ciudad de Santo Domingo de los Tsáchilas, con pacientes adultos mayores, desde el dia uno de diciembre de dos mil catorce (1/12/14) hasta el dia veinte y siete de marzo de dos mil quince $(27 / 3 / 15)$.

Es cuanto puedo certificar en honor a la verdad y autorizo a los investigadores hacer uso del presente para los fines pertinentes.

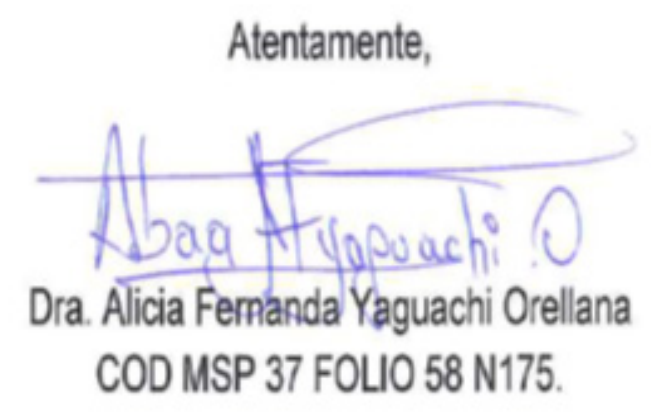

Santo Domingo, 27 de marzo de 2015 


\section{Anexo 5. Prescripción médica del ejercicio}

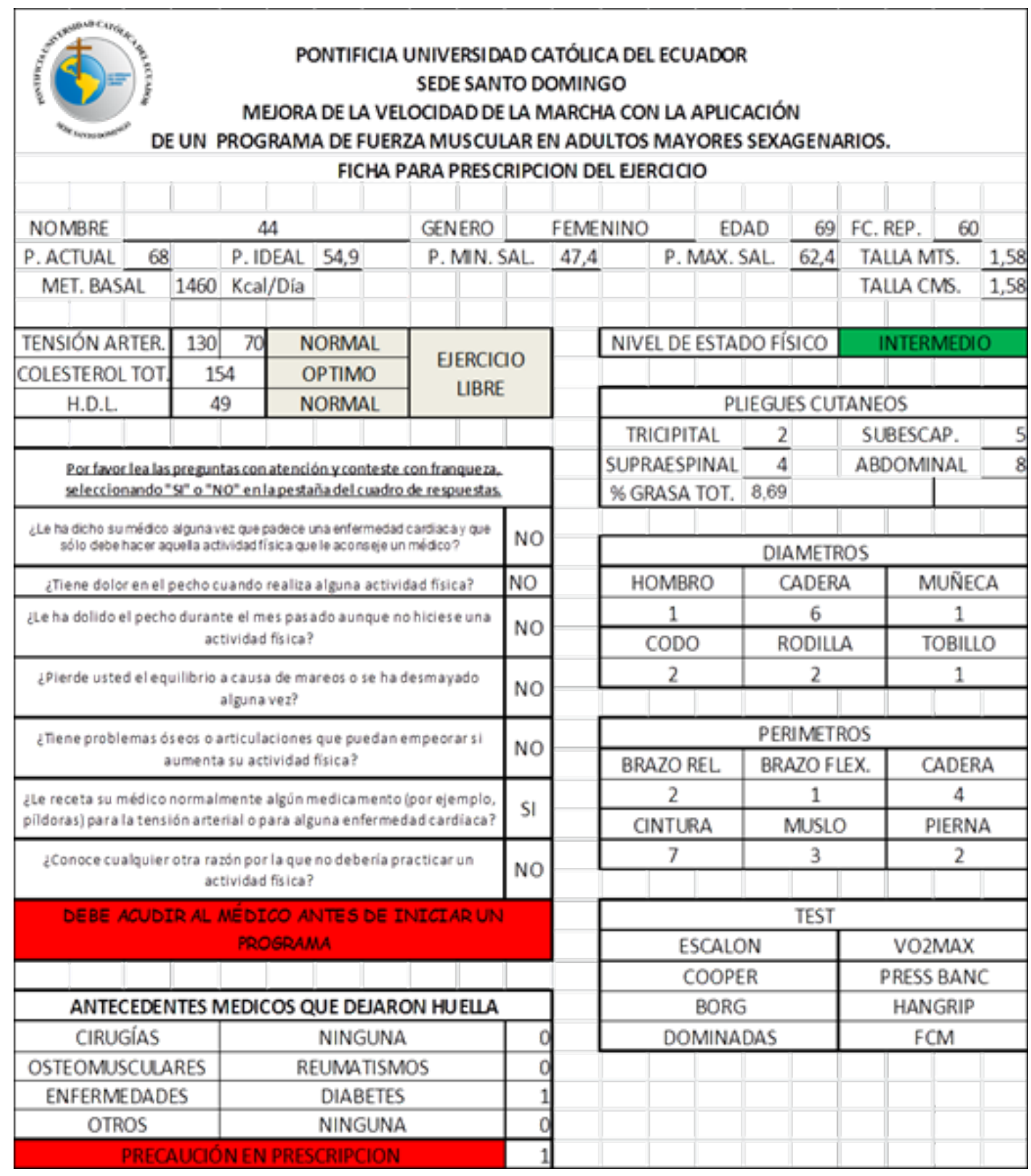


Anexo 6. Valoraciones de los adultos mayores de Santo Domingo de los Tsáchilas
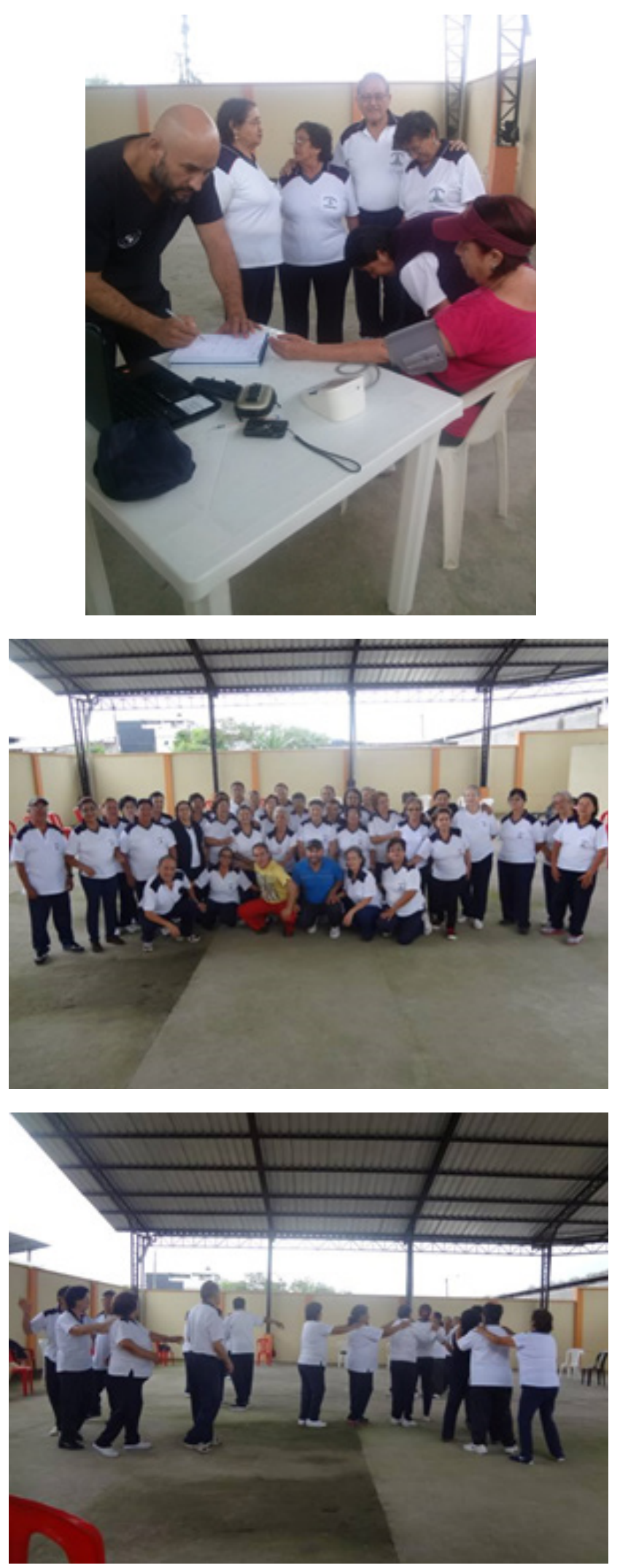

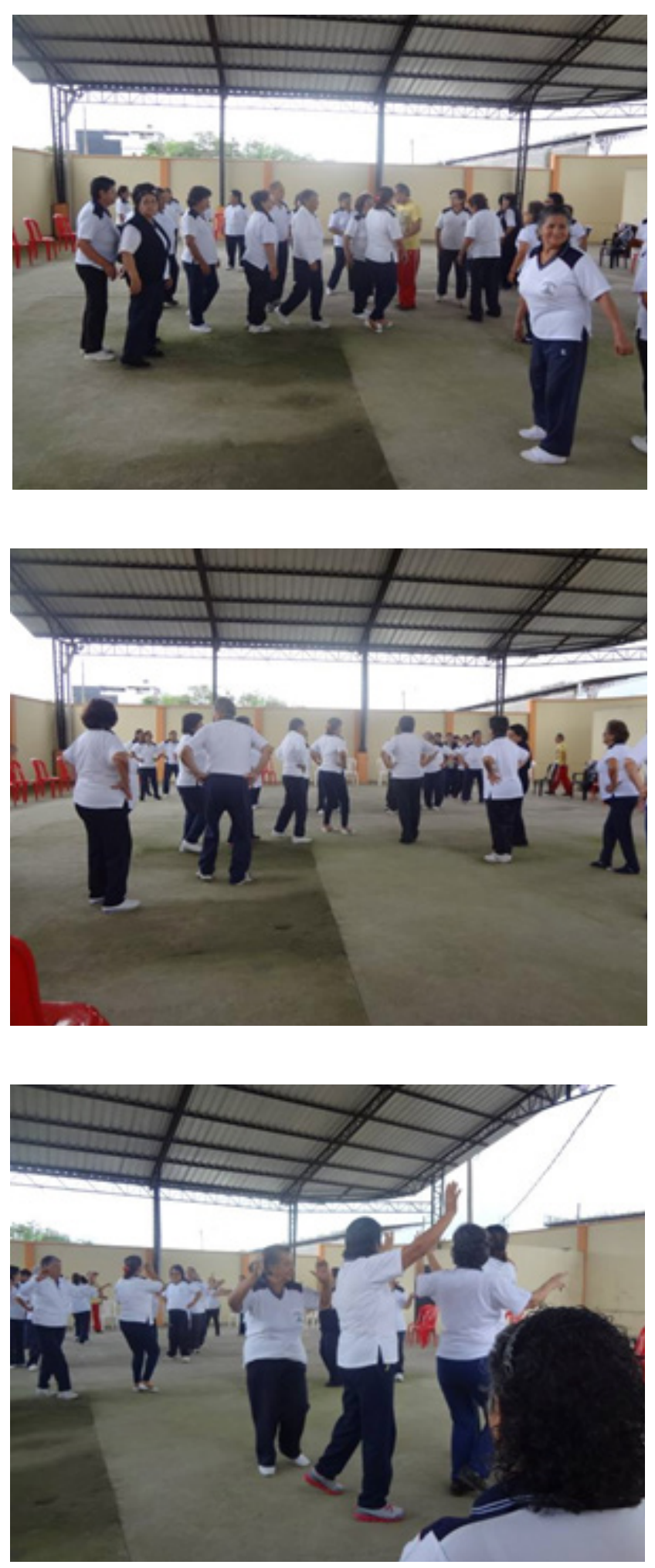